\title{
Management of Cytopenias During Treatment with Lenalidomide in Patients with Myelodysplastic Syndromes
}

\author{
Minoo Battiwalla ${ }^{1}$ and Richard A. Wells ${ }^{*}, 2$ \\ ${ }^{1}$ Roswell Park Cancer Institute, Buffalo, NY, USA \\ ${ }^{2}$ Sunnybrook Health Sciences Centre, 2075 Bayview Ave., Room T2 012, Toronto, ON M4N 3M5, Canada
}

\begin{abstract}
Lenalidomide is approved for the treatment of Low and Intermediate-1 risk International Prognostic Scoring System categorized myelodysplastic syndromes (MDS) with deletion (del (5q)), and has an evolving role in other subtypes of MDS. Severe cytopenias may emerge with lenalidomide treatment, which appear to be related to elimination of the dysplastic clone. Although guidelines based on expert opinion have been published, the optimum management of lenalidomide-induced cytopenias in MDS in the "real world" setting is still evolving. We report two illustrative cases of lenalidomide-induced cytopenias in patients with del (5q) MDS, present an updated review of the literature and provide practical guidance for the management of cytopenias during lenalidomide treatment of MDS.
\end{abstract}

Keywords: Lenalidomide, MDS, cytopenias.

\section{INTRODUCTION}

Myelodysplastic syndromes (MDS) comprise a spectrum of clonal hematopoietic stem cell disorders characterized by ineffective hematopoiesis and a tendency towards transformation to acute myeloid leukemia (AML)[1]. Lenalidomide (Revlimid $^{\circledR}$; Celgene Corporation, Summit, NJ), is an oral immunomodulatory agent approved by the U.S. Food and Drug Administration for the treatment of patients with transfusion-dependent anemia due to Low- or Intermediate-1-risk MDS associated with a deletion (del $(5 q))$ cytogenetic abnormality with or without additional cytogenetic abnormalities [2]. In the registration trial of lenalidomide in Low- or Intermediate-1-risk MDS with a $\operatorname{del}(5 q), 67 \%$ of patients achieved transfusion independence after a median of 4.6 weeks of treatment [3]. In a separate study of Low to Intermdiate-1 risk MDS patients without del(5q), transfusion independence was seen in $17 \%$ of patients receiving lenalidomide [4] (Table 1).

The main adverse events associated with lenalidomide treatment are neutropenia and thrombocytopenia [3, 4]. These cytopenias occur early, at a median of three weeks, suggesting an impact on committed hematopoietic progenitors. However, peripheral blood progenitor mobilization for autologous transplant in myeloma is compromised by prolonged lenalidomide, suggesting a cumulative impact on earlier hematopoietic precursors, perhaps by an unrelated mechanism [5, 6]. The incidence of neutropenia/thrombocytopenia depends on the disease being treated: treatment-emergent grade III-IV neutropenia occurred in $21 \%$ of myeloma, $53 \%$ of del (5q) MDS and

*Address correspondence to this author at the Sunnybrook Health Sciences Centre, 2075 Bayview Ave., Room T2 012, Toronto, ON M4N 3M5, Canada; Phone: 416-480-5700 Fax: 416-480-6002

Email: richard.wells@sunnybrook.ca
$30 \%$ of non-del (5q) MDS and grade III-IV thrombocytopenia occurred in $20 \%$ of myeloma, $50 \%$ of del (5q) MDS and $25 \%$ of non-del (5q) MDS [3-5].

The elegant study by Sekeres et al. demonstrated that treatment-emergent lenalidomide-induced cytopenias correlate to eventual clinical benefit [6]. However, despite the publication of expert consensus guidelines [7], clear examples of how to manage treatment-related cytopenias in "real world" clinical practice while continuing lenalidomide therapy are lacking. Here we present two illustrative cases regarding a rational approach to the management of treatment-related cytopenias during lenalidomide therapy in patients with MDS.

\section{CASE 1}

A 63-year-old woman was referred for evaluation of asymptomatic macrocytic anemia in July, 2005. A hemogram performed at the time of evaluation showed that her hemoglobin $(\mathrm{Hb})$ was $102 \mathrm{~g} / \mathrm{L}$, mean corpuscular volume (MCV) was $122 \mathrm{fL}$, white blood cell count (WBC) was $3,300 / \mathrm{mm}^{3}$, absolute neutrophil count (ANC) was $1,300 / \mathrm{mm}^{3}$, and platelet count was $325,000 / \mathrm{mm}^{3}$ (Fig. 1). Renal function was normal and the serum erythropoietin level was elevated (594 U/L). Bone marrow aspiration revealed increased cellularity, $4 \%$ blasts, dysplastic erythropoiesis, and an increased number of megakaryocytes, which frequently had small mononuclear and hypolobated forms. Metaphase cytogenetics showed 46XX, $\operatorname{del}(5)(\mathrm{q} 15 ; \mathrm{q} 33)$ in 5 of 11 metaphases.

The patient's anemia progressed and she eventually required blood transfusions. Between March 2006 and February 2007, she received a total of 19 units of packed red blood cells (PRBC). Her disease course was complicated in October 2006 by a non-ST-elevation myocardial infarction. 
Table 1. Lenalidomide Activity in Low/Int-1 Risk MDS

\begin{tabular}{|l|l|l|}
\hline \multicolumn{1}{|c|}{ Parameters } & \multicolumn{1}{|c|}{ del(5q) MDS [3] } & Non del(5q) MDS [4] \\
\hline \hline Efficacy & $67 \%$ & $26 \%$ \\
\hline RBC transfusion independence & & $17 \%$ \\
\hline Hematologic improvement by IWG 2006 criteria & $5.4 \mathrm{~g} / \mathrm{dL}$ & $3.2 \mathrm{~g} / \mathrm{dL}$ \\
\hline Median rise of hemoglobin & $>12$ months & 41 weeks \\
\hline Median duration of transfusion independence & 3 months & 4 months \\
\hline Time to transfusion independence in $90 \%$ of responders & $(45+28) \%$ & $(9+11) \%$ \\
\hline Cytogenetic responses (complete or $>50 \%$ reduction) & & \\
\hline Safety & $53 \%$ & $55 \%$ \\
\hline Grade 3 or 4 neutropenia & $50 \%$ & $44 \%$ \\
\hline Grade 3 or 4 thrombocytopenia & \\
\hline
\end{tabular}

(A)

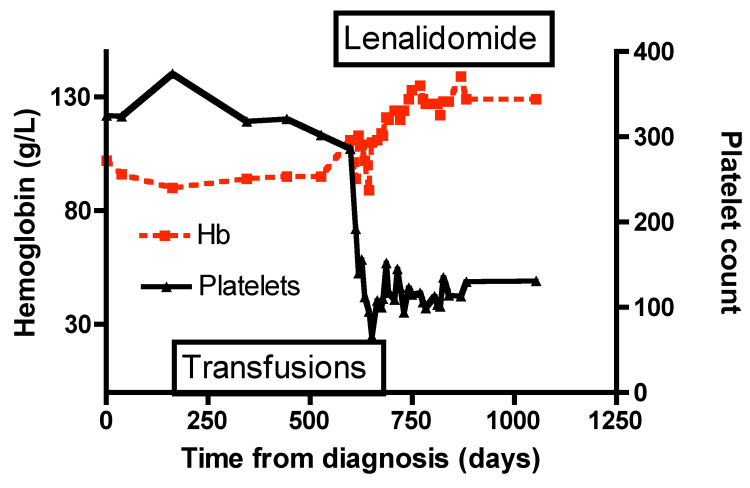

(B)

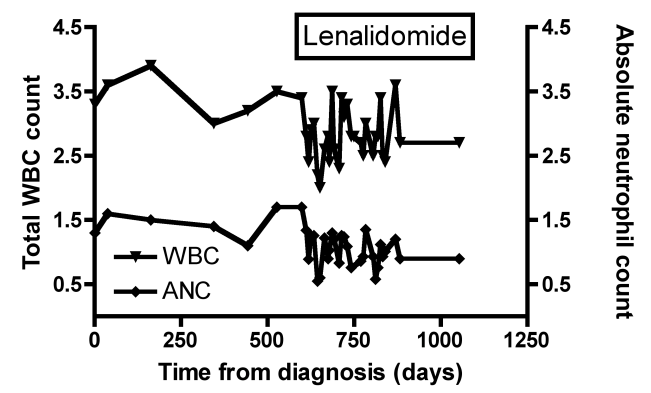

Fig. (1). Case 1, in which lenalidomide treatment was initiated in February 2007. Graph A shows course of $\mathrm{Hb}$ (pink line) and platelet count (dark blue line). Graph B shows ANC (pink line) and WBC (dark blue line).

In January, 2007, the patient was receiving regular blood transfusions at a rate of 2 units of PRBC every four weeks. Physical examination was unremarkable and a hemogram indicated that the $\mathrm{Hb}$ level was $95 \mathrm{~g} / \mathrm{L}, \mathrm{MCV}$ was $104 \mathrm{fL}$, WBC was $3,500 / \mathrm{mm}^{3}$, ANC was $1,700 / \mathrm{mm}^{3}$, and the platelet count was $302,000 / \mathrm{mm}^{3}$. However, there was evidence of secondary hemochromatosis; the serum ferritin level was $1174 \mathrm{pmol} / \mathrm{L}$, and transferrin saturation was 94\%. Serum electrolytes were normal and the serum creatinine level was $79 \mu \mathrm{mol} / \mathrm{L}$. A repeat bone marrow aspiration showed no progression.
Treatment with oral lenalidomide was initiated at the standard dose of $10 \mathrm{mg}$ given once daily for 21 days of each 28-day cycle. Six weeks after starting lenalidomide, the patient achieved transfusion independence. She experienced no adverse effects, with the exception of scalp pruritus, which resolved spontaneously within 48 hours. Platelet count decreased rapidly after treatment initiation and then remained at levels between $100,000 / \mathrm{mm}^{3}$ and $150,000 / \mathrm{mm}^{3}$. The WBC and ANC remained relatively stable at approximately $3,000 / \mathrm{mm}^{3}$ and $1,000 / \mathrm{mm}^{3}$, respectively. Repeat cytogenetic analysis performed after completion of six cycles of lenalidomide showed persistence of the del (5q) in 20/20 metaphases despite the complete erythroid response.

\section{CASE 2}

In March 2007, a 72-year-old woman was referred for evaluation of unexplained macrocytic anemia that had persisted for 12 months. During that period, she had received a total of 14 units of packed RBCs. Her hemogram showed an $\mathrm{Hb}$ level of $10.4 \mathrm{~g} / \mathrm{dL}, \mathrm{MCV}$ of $94.3 \mathrm{fl}$, WBC of $4,100 / \mathrm{mm}^{3}$, ANC of $2,300 / \mathrm{mm}^{3}$, and platelet count of $571,000 / \mathrm{mm}^{3}$ (Fig. 2). Her renal function was normal. Bone marrow aspiration results were consistent with $5 \mathrm{q}-$ syndrome, and 20 of 20 metaphases exhibited $\operatorname{del}(5 q)(q 13 ; q 33)$.

Treatment was initiated with oral lenalidomide $10 \mathrm{mg}$ given once daily for 21 days of each 28-day cycle. The patient had a brisk erythroid response and became transfusion-independent after the first cycle of treatment. The patient experienced mild muscle cramping in her hands and legs. Moderate scalp pruritus resolved with diphenhydramine and moderate to severe diarrhea was controlled with loperamide. The patient's platelet count declined steeply during the first two treatment cycles, reaching a nadir of $81,000 / \mathrm{mm}^{3}$ in the second cycle. Her ANC decreased to $700 / \mathrm{mm}^{3}$ at the beginning of cycle 2 , but rebounded following a brief course of granulocyte colonystimulating factor (G-CSF) support (300 $\mu \mathrm{g}$ given subcutaneously twice weekly for four doses); thereafter, the ANC remained above $1,000 / \mathrm{mm}^{3}$. Repeat cytogenetic analysis performed after completion of ten cycles of lenalidomide revealed the presence of the $\operatorname{del}(5 \mathrm{q})(\mathrm{q} 13 ; \mathrm{q} 33)$ 
in 20/20 metaphases despite the achievement of durable transfusion independence.

(A)

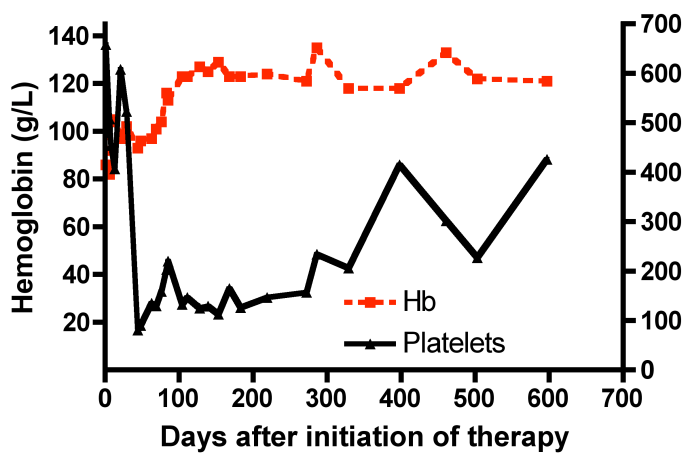

(B)

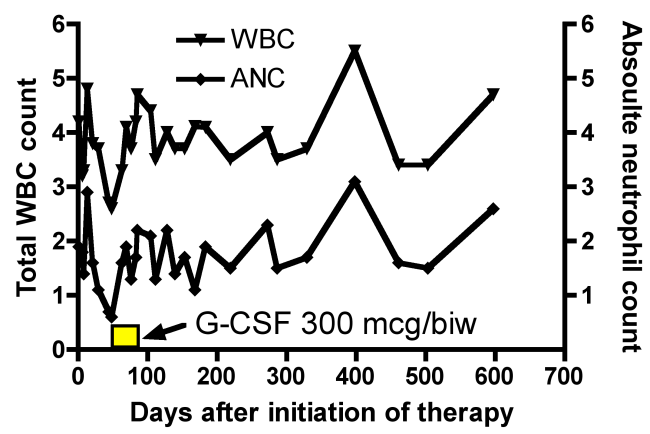

Fig. (2). Case 2, in which lenalidomide treatment was initiated in March 2007. Graph A shows course of $\mathrm{Hb}$ (pink line) and platelet count (dark blue line). Graph B shows ANC (pink line) and WBC (dark blue line).

\section{DISCUSSION}

The availability of lenalidomide has revolutionized the management of del $(5 q)$ MDS. However, the use of lenalidomide in patients with this disease is commonly associated with clinically significant and very dramatic neutropenia and thrombocytopenia. Although del (5q) is the single most common cytogenetic abnormality in MDS, nonetheless del (5q) MDS is a rare disease in absolute terms, and the great majority of practicing hematologists will not have the opportunity to gain broad direct clinical experience and comfort in dealing with lenalidomide and its adverse effects. As a consequence, there is a tendency to undertake premature or excessive dose reductions or even to abandon lenalidomide therapy altogether in this patient group; thus, the dramatic response rates seen in clinical trials may not translate readily to everyday clinical practice. In this context, it may be of significant value for clinicians to learn from descriptions of the experience of clinical management of del (5q) MDS patients in the practice setting. In this paper we describe the management of lenalidomide-induced cytopenias in two "real world" del (5q) MDS patients, both of whom went on to benefit significantly from their treatment.

Management of lenalidomide-induced cytopenias requires an individualized approach tailored to the specific condition of the patient. Options include treatment delays or discontinuation, dose reductions, or growth factor support [2, 7]. In both of the cases presented here, the initiation of lenalidomide therapy resulted in a rapid and significant decrease in platelet counts. While one patient required a short course of granulocyte-colony stimulating factor (GCSF), both patients were able to continue lenalidomide therapy and eventually achieved transfusion independence.

The optimum initial dosing for lenalidomide remains a matter of debate. Initial dosing recommendations for lenalidomide were identified by classical phase I/II doseescalation/de-escalation trials with safety as the major determinant. These doses are $10 \mathrm{mg}$ once daily and $10 \mathrm{mg}$ on the first 21 days of a 28-day cycle. As the optimum dosing for therapeutic benefit has not been precisely defined and as safety is the overriding concern in chronic disorders, higher initial doses are not recommended. Although lower starting doses have been found efficacious in MDS, it is not established whether lower dose schedules are equally beneficial, less cytopenic or require a different schema of safety monitoring.

Lenalidomide is predominantly eliminated renally and consequently renal dysfunction significantly raises lenalidomide exposure. As renal impairment progresses to moderate, severe, or end-stage renal disease, the area under the concentration-time curve increases $\sim 185 \%$ to $420 \%$, and $\mathrm{t}_{1 / 2}$ is prolonged by approximately 6 to 12 hours [8]. Guidance for dose adjustment in renal insufficiency is provided in a recent update in the package insert [2]. Interestingly, baseline renal dysfunction does not have a significant impact on achieving transfusion independence or transformation to AML [9].

Moderate-to-severe neutropenia and thrombocytopenia are expected soon after the initiation of lenalidomide therapy in most patients. In the registration trial, grade 3 or 4 neutropenia and thrombocytopenia occurred in 55\% and $44 \%$ of patients, respectively, and $62 \%$ of treatment-related cytopenias were reported within the first 8 weeks [3]. In patients who developed grade 3 or 4 neutropenia, the median time to onset was 42 days, and the median time to documented recovery was 17 days [3]. In patients who developed grade 3 or 4 thrombocytopenia, the median time to onset was 28 days, and the median time to documented recovery was 22 days. Therefore, weekly monitoring of blood cell counts is recommended for MDS patients during the first 8 weeks of lenalidomide therapy and at least monthly thereafter.

The development of treatment-related cytopenias particularly a significant reduction in platelet counts - has been linked to the achievement of transfusion independence [6]. An association was seen only in patients with del( $5 q)$, indicating that a direct cytotoxic effect of lenalidomide specific to the del $(5 q)$ clone may correlate with transfusion independence. Indeed, the frequent and early onset of cytopenias is consistent with the suppressive effect of lenalidomide on the del $(5 q)$ clone. These findings suggest that efforts to continue lenalidomide therapy while effectively managing cytopenias may provide the best chance of achieving favorable clinical outcomes. While a definitive survival trial for lenalidomide versus placebo in the treatment of MDS is unlikely to be performed, a comparison of the survival of $\operatorname{del}(5 \mathrm{q})$ patients treated with 
lenalidomide to historical controls could conceivably result in earlier use of lenalidomide in $\operatorname{del}(5 \mathrm{q}) \mathrm{MDS}$ and greater tolerance of the inevitable cytopenias.

It is unclear whether lenalidomide can be given safely to patients with existing neutropenia and/or thrombocytopenia prior to the start of therapy. An expert panel noted that, in their opinion, lenalidomide therapy can still be given to patients presenting with a neutrophil count less than $1,000 / \mathrm{mm}^{3}$, provided that G-CSF is co-administered [7]. Regarding the question of whether patients with baseline thrombocytopenia (platelet count less than $25,000 / \mathrm{mm}^{3}$ ) should received lenalidomide therapy, the expert panel was mixed: some felt that lenalidomide could be given with platelet support, based on the assumption that the thrombocytopenia is likely to resolve once a response has been achieved, whereas others felt that lenalidomide should not be given to patients with platelet counts less than $50,000 / \mathrm{mm}^{3}$ prior to treatment due to the risk of worsening thrombocytopenia, regardless of erythroid response [7]. In the registration trial, baseline thrombocytopenia was the most important variable associated with a reduced probability of achieving transfusion independence or a cytogenetic response [3]. It was noted that the number of consecutive days of drug treatment was significantly lower among patients with baseline thrombocytopenia due to treatment interruptions for myelosuppression, which suggests that the duration of lenalidomide treatment is a determinant of clonal suppression and hematologic improvement. No dose adjustment in the starting dose of lenalidomide is required for advanced age: a subanalysis of the study involving lenalidomide for $\operatorname{del}(5 q)$ MDS found no difference in the incidence of grade III or higher cytopenias in patients $>75$ years $(76 \%)$ versus $\leq 75$ years $(84 \%)$ [10].

In summary, the management of treatment-related cytopenias that emerge during treatment with lenalidomide in patients with Low- or Intermediate-1-risk MDS and $\operatorname{del}(5 \mathrm{q})$ should be tailored to the individual patient, with adjustments to the starting dose for renal function but not for age. Emphasis is placed on avoiding treatment delays by dose adjustments and judicious myeloid growth factor support, so that optimal clinical outcomes may be achieved.

\section{ACKNOWLEDGMENTS}

The authors received editorial support in the preparation of this manuscript from Excerpta Medica, funded by Celgene. The authors were fully responsible for content and editorial decisions for this manuscript.

\section{REFERENCES}

[1] Battiwalla M, Young N. Myelodysplastic Syndromes. In: Young, Rodgers, Eds. Bethesda: Handbook of Hematology 2005.

[2] Celgene_Corporation. Revlimid® (lenalidomide). Official prescribing information. January, 2010.

[3] List A, Dewald G, Bennett $\mathrm{J}$, et al. Lenalidomide in the myelodysplastic syndrome with chromosome $5 \mathrm{q}$ deletion. $\mathrm{N}$ Engl J Med 2006; 355: 1456-65.

[4] Raza A, Reeves JA, Feldman EJ, et al. Phase 2 study of lenalidomide in transfusion-dependent, low-risk, and intermediate1 risk myelodysplastic syndromes with karyotypes other than deletion 5q. Blood 2008; 111:86-93.

[5] Kumar S, Giralt S, Stadtmauer EA, et al. Mobilization in myeloma revisited: IMWG consensus perspectives on stem cell collection following initial therapy with thalidomide-, lenalidomide-, or bortezomib-containing regimens. Blood 2009; 114: 1729-35.

[6] Sekeres MA, Maciejewski JP, Giagounidis AA, et al. Relationship of treatment-related cytopenias and response to lenalidomide in patients with lower-risk myelodysplastic syndromes. J Clin Oncol 2008; 26: 5943-9.

[7] Giagounidis A, Fenaux P, Mufti GJ, et al. Practical recommendations on the use of lenalidomide in the management of myelodysplastic syndromes. Ann Hematol 2008; 87: 345-52.

[8] Chen N, Lau H, Kong L et al. Pharmacokinetics of lenalidomide in subjects with various degrees of renal impairment and in subjects on hemodialysis. J Clin Pharmacol 2007; 47: 1466-75.

[9] Battiwalla M, Fu T, Knight RD, et al. Impact of Baseline Renal Function on Transfusion-Independence Response, Survival, and Transformation to Acute Myeloid Leukemia in Patients with Deletion 5q: A Sub-Analysis of the MDS-003 Study. ASH Ann Meet Abst 2008; 112: 5088-

[10] Battiwalla $\mathrm{M}, \mathrm{Fu} \mathrm{T}$, Knight $\mathrm{RD}$, et al. Impact of Age on Transfusion Independence Response, Survival, and Transformation to Acute Myeloid Leukemia in Patients with Deletion 5q: A SubAnalysis of the MDS-003 Study. ASH Ann Meet Abst 2008; 112: 5071. 